\title{
Implications of World Peak Population for Canada
}

\author{
Alain Bélanger \\ Barry Edmonston ${ }^{1}$
}

\section{Introduction}

The last century witnessed the fastest population growth in human history, but this is now ending. ${ }^{2}$ Recent 2017 United Nations population projections assume that current fertility levels of 2.5 children per woman will decrease to 2.2 in 2050 and to a below-replacement level of 1.99 in 2100 (United Nations 2017). ${ }^{3}$ Estimated at 7.2 billion in 2013, the world's population is expected to continue to grow, but at a much slower pace. It will eventually peak at slightly more than 11 billion in the 22nd century, and thereafter slowly decrease for the first time in several centuries. Under the United Nations' low-fertility assumption, world population would peak even sooner, at 8.7 billion in about 2050 and decrease to 7.3 billion by 2100 .

Today's current population is increasing by 1.1 per cent per year, compared to almost 2 per cent as recently as 1970-75. By the end of the current century, in 2095-2100, the United Nations expects world population growth to decrease to 0.1 per cent per year; however, peak population size does not occur in this century, according to the 2017 United Nations' medium-fertility population projections. By 2095-2100, world population growth is small but still positive. If the United Nations' rates are extrapolated beyond 2100, world population growth would decrease to zero in about 2110 (by linear extrapolation) or 2115 (by exponential extrapolation). Even though world fertility is projected to fall below replacement levels by 2100 , the younger age distribution in some high-fertility countries provides "momentum" for continued population growth past 2100 . Nevertheless, global world population growth is expected to cease by 2075 in all regions except Africa and Oceania.

1. Alain Bélanger, Urbanisation Culture Société Research Centre, L'Institut national de la recherche scientifique (INRS), Montréal, QC H2X 1E3, email: alain.belanger@ucs.inrs.ca; and Barry Edmonston, Department of Sociology and Population Research Group, Box 3050 Stn CSC, University of Victoria, Victoria, BC V8W 3P5, email: be@uvic.ca.

2. We prepared an earlier draft of this report in response to a request from the Social Sciences and Humanities Research Council (SSHRC) for a brief examination of the implications of global peak population on Canada's future. SSHRC initially discussed the idea of this report with Professor Roderic Beaujot, Director of the Strategic Knowledge Cluster on Population Change and Lifecourse at Western University. He conferred with Alain Bélanger and Barry Edmonston, who agreed to serve as editors of the report. Authors were then asked to write sections of the report dealing with six key questions. WeBélanger and Edmonston thank Rod Beaujot, Sharon Lee, and Frank Trovato for reading previous drafts and providing thoughtful and helpful comments. The authors have revised and updated the previous SSHRC report for publication in Canadian Studies in Population.

3. Replacement-level fertility is about 2.1 children born to a woman over her lifetime, taking into account the effects of low mortality and the sex ratio of 105 male births per 100 female births. 
There are large variations in current fertility levels across countries and regions. Although only 8 per cent of the world's population lives in countries with high fertility (5 or more births per woman), they account for more than one-third of the world's annual population increase. Of the 22 countries with high fertility, the United Nations' population projection (2017: xxiv) expects no country to experience fertility with 5 or more births per woman by 2050 . Most of the world's current population (46 per cent) lives in intermediate-fertility countries, with fertility between 2.1 and 5 births per woman over her lifetime. Only one-third of the world's population is expected to be in this category by 2050, with the remainder achieving low fertility. The other large group of the world's population (46 per cent) already lives in low-fertility countries that have less than 2.1 births per woman. These countries include all of Europe and North America, plus 19 countries in Asia, 15 in Latin America and the Caribbean, and 5 in other regions. By 2050, the United Nations expects that 69 per cent of the world's population will reside in low-fertility countries.

National population growth varies greatly. There will still be major growth for several decades in a small number of countries, such as Nigeria and India. But there are currently 75 countries with below-replacement fertility_-including Canada_ and all will eventually decrease without counterbalancing net immigration. More than a dozen countries in Eastern Europe and Russia will experience large population declines in coming decades, due to very low fertility levels and emigration. Global trends will affect the role of migration on population, with declines in the pool of potential immigrants from many source countries.

For several decades following World War II, Canada's demographic growth was strong. Canadians had many babies and welcomed many immigrants. But in the 1970s fertility fell to below-replacement levels. Natural increase, the difference between the number of births and the number of deaths, is still positive due to population momentum, but is declining and will turn negative in the future. Net immigration thus represents an increasing share of population change. The demographic opportunity window - which created a younger population with a higher proportion of adults in the working ages - is closing for Canada, as baby boomers retire from the labour market. Assuming continuing moderate immigration, Statistics Canada's 2013 population projections (Statistics Canada 2015) indicate that the population will increase from 35.2 million in 2013 to 51.0 million in 2063 , under medium-growth assumptions. But immigration has small effects on population aging and the proportion 65 years and older could increase from 15 per cent now to between 24 and 28 per cent in 2063. Under Statistics Canada's medium-growth population projections, population growth will drop from 1.1 per cent annual growth at present to 0.7 per cent in 2063 .

\section{Interpreting peak population}

The notion of the world's peak population is a special aspect of demographic transition theory, which was originally proposed by Princeton University demographer Frank Notestein in 1945 (demographic transition theory and later refinements are discussed in Kirk 1996). Notestein suggested that the demographic transition involved four stages. Stage I characterized pre-industrial societies with high birth rates and high, fluctuating death rates. These societies experienced negligible long-term population growth and had young age structures. Stage II occurred when death rates began to fall with improving public health and standard of living. Population growth increases and the age structure becomes slightly younger. In Stage III, birth rates began to decline, with slackening of population growth and population structure becoming older. Stage IV is reached when birth and death rates both become low. Population growth becomes negligible and there is an old age structure. 
From the perspective of the demographic transition, the world's peak population occurs in Stage IV, when global fertility levels decrease to (or below) replacement-level, which creates birth and death rates that are relatively equal. At this point, population growth slackens and becomes close to zero, the world population stabilizes (or begins to decrease), and there is an older age structure. This means that the concept of world peak population involves three distinct outcomes: (1) population growth ceases and may, depending upon relative birth and death rates, become negative; (2) the size of the world's population peaks, and remains constant or may decrease if fertility is below replacement level; and (3) the overall age structure of the world's population becomes older. These three demographic consequences are associated with the idea of a peak population, and are discussed in more detail in the Canadian context in the papers in this special issue of Canadian Studies in Population.

\section{Population growth}

Fifty years ago, the global population was growing at almost 2 per cent per year (see Figure 1). Today, it is growing by 1.1 per cent per year. Although the world's population is expected to continue growing until the end of the 21 st century, according to the United Nations medium-fertility assumptions, the rate at which this growth will occur is expected to continue to fall. In recent years, the population of Africa has had the fastest growth among all regions, increasing at a rate of 2.6 per cent annually in 2010-15; however, this rate is beginning to fall and is projected to reach 1.8 in 2045-50 and 0.7 in 2095-2100. Population growth will fall to zero by 2050 for Europe, Asia, and Latin America and the Caribbean. Fertility will be below-replacement for North America, but net immigration will maintain positive population growth for the remainder of the 21 st century.

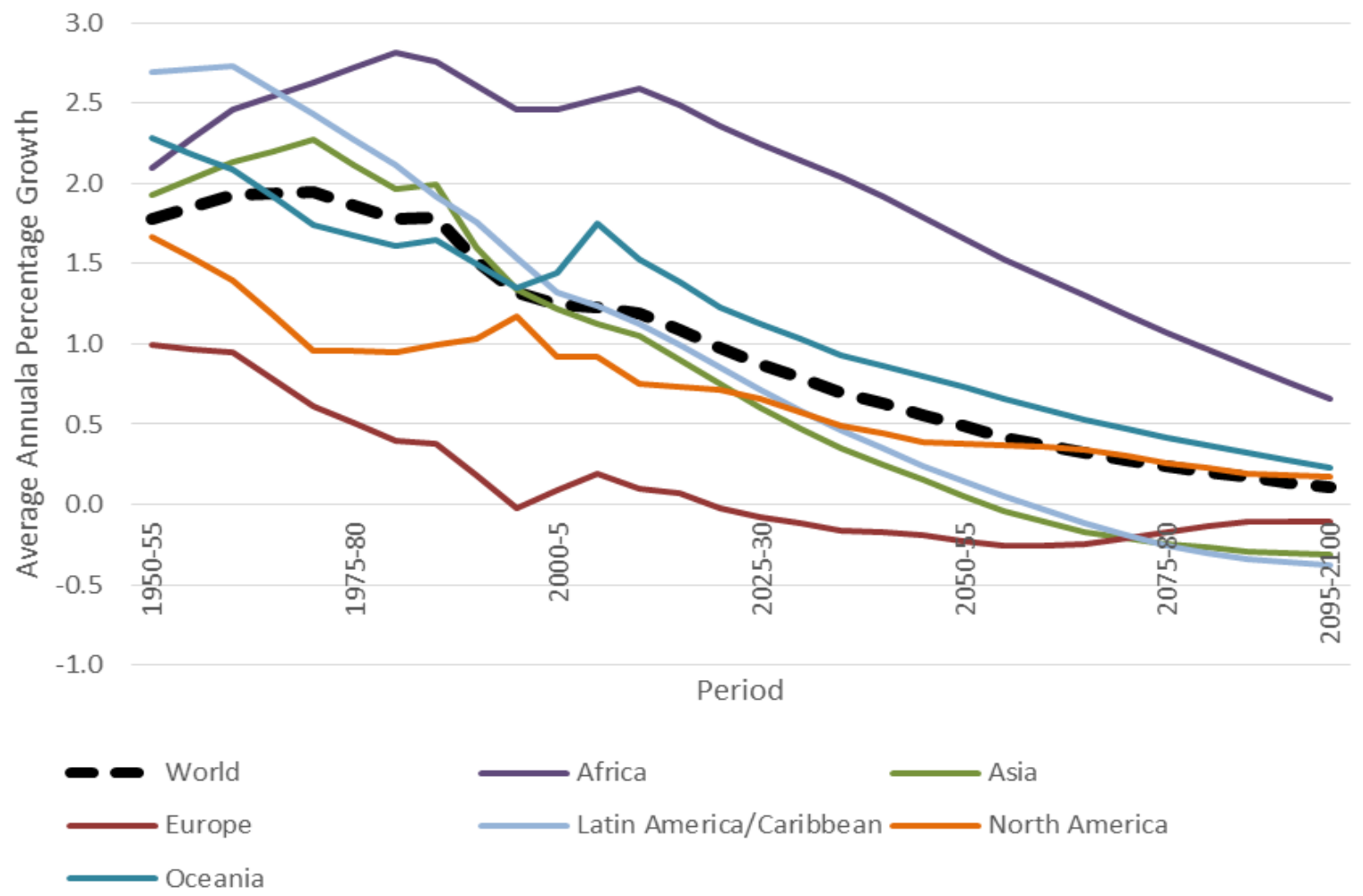

Figure 1. Average annual rate of population change by region: Estimates and medium variant population projections, 1950-2100.

Source: United Nations 2017: Table A5. 


\section{Population size}

The world's population currently continues to grow, but more slowly than in the past. The world's population is projected (using United Nations medium-fertility assumptions) to increase by slightly more than one billion people over the next 13 years, reaching 8.6 billion in 2030, and to increase further to 9.8 billion in 2050 and 11.2 billion by 2100 . As shown in Figure 2, more than half of the anticipated growth in global population between now and 2050 is expected to occur in Africa. Of the additional 2.2 billion people who will be added between 2017 and 2050, 1.3 billion will be in Africa. Asia is expected to be the second-largest contributor to this future growth, adding over 750 million people between 2017 and 2050. Population growth in Africa and Asia is followed by Latin America and the Caribbean, Northern America, and Oceania, where growth is projected to be more modest. In the medium-fertility population projection, Europe will be the only region with a smaller population in 2050 than in 2017. From 2050 to 2100, Africa will be the main contributor to global population growth.

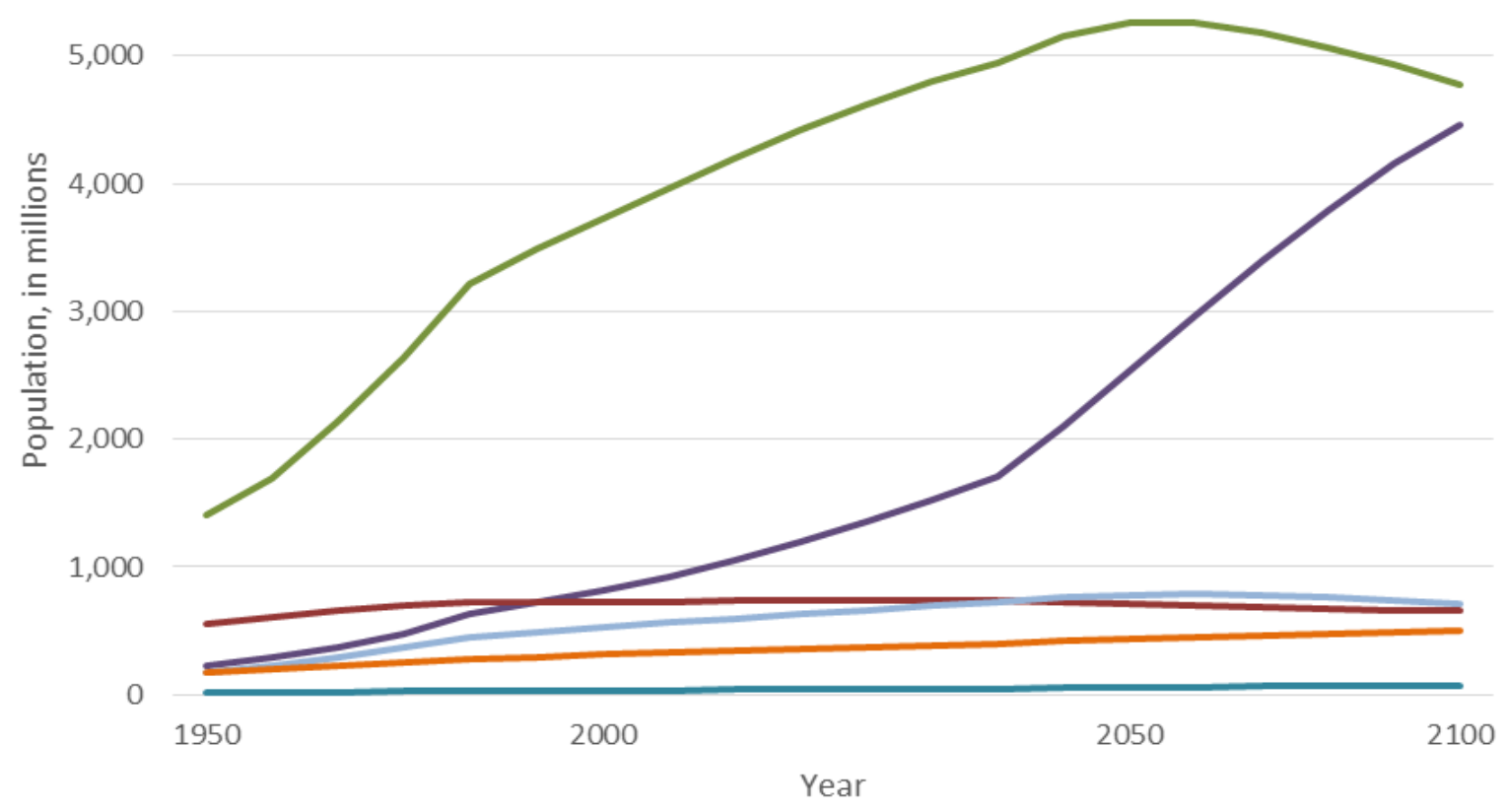

-Africa —Asia —-Europe Latin America/Caribbean — North America — Oceania

Figure 2. Population size by region: Estimates and medium variant population projections, 1950-2100. Source: United Nations 2017: Table A1.

\section{Age structure}

As fertility declines and life expectancy rises, the proportion of the population in older ages increases relative to the population in younger ages (see Figure 3). This phenomenon, known as population aging, now occurs throughout the world. In 2017, there are an estimated 960 million people aged 60 or over in the world, comprising 13 per cent of the global population. The population aged 60 or above is growing at a rate of about 3 per cent per year, almost three times the overall global population growth rate. Rapid population aging will occur in all parts of the world, and by 2050 all regions of the world except Africa will have one-fourth or more of their populations aged 60 and above. The number of older persons in the world is projected to be 1.4 billion in 


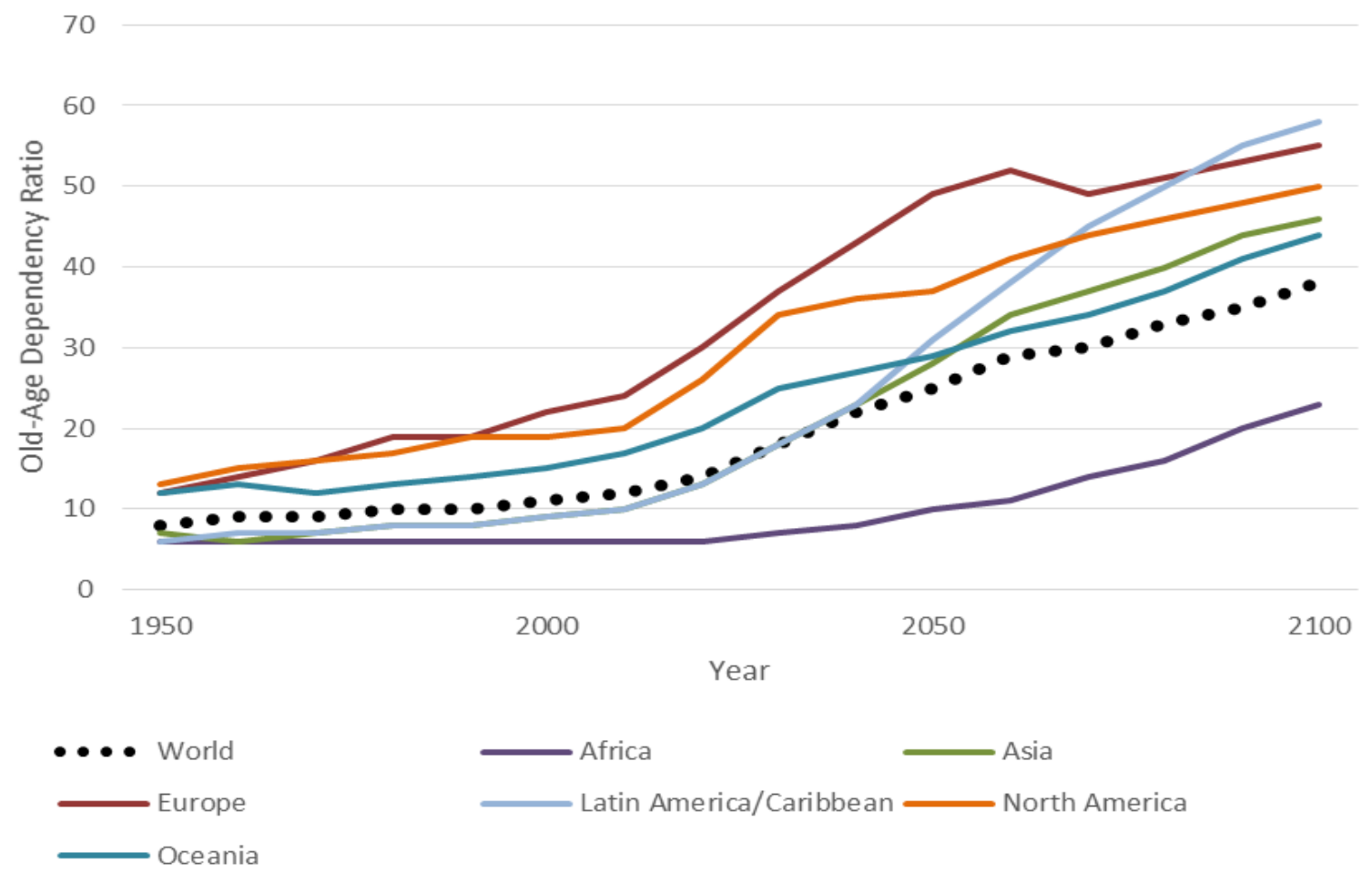

Figure 3. Old-age dependency ratios by region: Estimates and medium variant population projections, 1950-2100.

Source: United Nations 2017: Table A33.

Note: Old-age dependency ratio is defined as the number of people aged 65 years of age and older per 100 people aged 15 to 64 years.

2030, 2.1 billion in 2050, and 3.1 billion in 2100 - in other words, a tripling of the older population by the end of this century.

Population aging will have a dramatic effect on the elderly dependency ratio, defined as the number of person aged 65 or over per 100 persons aged 15-64 years. The elderly dependency ratio is a useful indicator as a ratio of the number of persons who are potential retirees or workers by virtue of their age. Currently, the elderly dependency ratio for the world is 14 , but is projected to increase to 25 in 2050 and 38 in 2100. In 2017, Africa has 6 persons aged 65 years or above for 100 persons aged 20 to 64 years. This ratio is 13 for Asia and for Latin America and the Caribbean, 20 for Oceania, 26 for Northern America, and 30 for Europe. By 2050, 7 countries in Asia, 24 in Europe, and 5 in Latin America and the Caribbean are expected to have potential support ratios around 50 . By the end of this century, in 2100 , the United Nations projects that elderly dependency ratios will be around 50 in all world regions except for Africa (which will be 23). These low values of 50 elderly per 100 workers-implying that there are roughly only two potential workers for every elderly person - underscore the fiscal and political pressures that many countries are likely to face in the coming decades in relation to public systems of health care, pensions, and social welfare for their growing older populations.

It should be noted that Notestein did not highlight the role of international migration in his original statement of the demographic transition, because his concern was with the relationship of modernization with birth and death dynamics. In later discussion, demographers noted that migration is likely to be involved in the demographic transition. Kingsley Davis (1963) argued that high population growth in Stage II of the demographic transition produces a multiphasic response, 
including outward migration when births greatly exceed deaths. Davis (1963: 352) contended that "under a prolonged drop in mortality...people found that their accustomed demographic behavior was handicapping them in their effort to take advantages being provided by the emerging economy." The multiphasic response to high population growth, according to Davis, is to postpone marriage, increase celibacy, resort to abortion, practice contraception, and emigrate overseas.

International migration cross-cuts the demographic transition for societies in two ways. During the demographic transition, there is a possibility that people emigrate from high population growth societies as part of the multiphasic response-as occurred in Western Europe in the 1800s when there were high emigration to Australia, Canada, the United States, and other destinations. And secondly, upon completion of the demographic transition - as will be the case for most countries in the coming century-international migration will subtract people from some countries, such as Eastern Europe, and lead to even larger population decreases or will add people to some below-replacement-level-fertility countries, such as Australia and Canada, and offset negative natural increase, which occurs when death rates exceed birth rates.

\section{Six key questions}

In the context of global population peaking, this Special Issue describes implications for Canada of global peak population for six key questions listed below, which are the focus for the following six papers. Each of the six papers addresses emerging issues, future challenges, and expanding our knowledge base for each key question.

Effectively nurturing the next generation is a critical topic for social policy. Nurturing the next generation involves establishing policies and programs for strengthening and training a new generation with the necessary knowledge and skills to be productive and contribute to changes to Canada's economy and society. This process depends greatly, however, on understanding the career aspirations and needs of youth and, at the same time, the employment demands for the future labour force.

What might Canadian families look like in the next 20 years, and how will they measure their well-being? The "traditional" two-parent, male-breadwinner, two-child family is already uncommon. Canadian family types have diversified in recent decades. Moreover, although some may yearn for a return to the "traditional" family, Canadian society does not appear to be worse off for evolving family trends.

Life cycle changes are challenging society, in Canada and the world. Demographic processes influence lifecourse and family cycles through their effects on longevity, parenting, and numbers and availability of spouses, children, parents, grandparents, and other relatives. Variations in lifecourse and family cycle-influenced as well by the number and composition of immigration-are key features in the frequency, direction, and timing of transitions such as entry into the labour force and retirement.

Global migration is primarily an urban phenomenon, with major effects on cities of the future, including Canadian cities. In 2010, about 214 million international migrants resided primarily in the large metropolises of a dozen immigrant-receiving countries. Over 63 per cent of Canada's immigrants live in Toronto, Vancouver, or Montréal, and immigrants will continue to gravitate to Canada's cities (Statistics Canada 2013). While immigration fuels Canadian cities' population and economic growth, cities have little control over national immigration policies or internal migration flows. In the future, Canadian cities will have more diverse and aging immigrants, and will be challenged to provide housing, jobs, education, and other social services, and promote the integration and inclusion of immigrants and their children. 
Changing demographics and migration also affect rural and remote communities, particularly in the North and the Arctic. With generally higher birth rates, Arctic populations are younger and growing. These populations are often also influenced by migration that dominates short-term changes for smaller population groups. For these rural and remote communities, population changes have implications for education, housing, healthcare, employment, and increased demand for resources such as water, electricity, and energy. Climate and ecological changes also have significant implications for these communities.

There are large potential effects of global peak population on Canada's energy, resource consumption, and climate change. Canada's foreseeable climate change is likely to include hotter summers and milder winters. Climate change will have direct and indirect effects on the environment, energy and resource demands, and public health across Canada. Canadians will find, for example, that greenhouse gases magnify the effects of air pollution in hotter summers and, in turn, increase human health risks.

\section{Global Peak Population: Emerging issues and future challenges}

Several overarching issues and challenges are addressed in the six papers. We do not summarize each of them but highlight some broad issues that are discussed in them.

\section{Labour force}

There will be important future challenges for Canada's labour force. While the Canadian population is aging, with shortages of labour in some remote communities and in some occupations, there are likely to be increasing challenges relating to youth and long-term unemployment. The growth in service sector employment and the related decline in manufacturing employment, as well as the effect of increased education levels on the workforce, will continue to affect the Canadian labour force. The nature of work has changed significantly over the last three decades, resulting in new issues, including the "hollowing-out" of middle-income employment, and the associated "hour-glass" economy. Higher-paid jobs are increasingly dependent on good education with skills, knowledge, and experience in selected occupations.

Even in these difficult economic times, some skilled workers-ranging from pipe fitters to chemical engineers - are in short supply and the world's leading companies compete fiercely for them. Unfilled vacancies continue in recent years, despite higher unemployment. Employers report that they have trouble filling some jobs, with technicians, salespeople, skilled trades, and engineers being the hardest to find. Northern and remote communities especially report the shortage of professionals and experienced technicians. Policymakers and educators face a key challenge to anticipate future changes in the demand for labour and to design policies that help Canadian workers to respond.

Canada's higher education system has expanded greatly during the past fifty years, with the training of a large number of students who have done relatively well. New challenges are emerging, with the need for a more diverse group of workers in a period of slower labour force growth. Moreover, these challenges will require more than simply expanding higher education. Attention will be needed to better design programs for early childhood education, changes in higher education that maintain broad participation while offering closer training for new employment opportunities, and expansion and improvement in lifelong learning programs, especially for older workers and recent immigrants. 


\section{Immigration}

Immigration will remain a major issue. Although difficult to predict and dependent on a number of factors, the number, characteristics, and integration of immigrants will be a key issue for policies and programs in coming decades. Besides the labour force aspects mentioned above, there are several important emerging issues related to immigration. The large numbers of immigrants who arrived in recent decades are, like other Canadians, becoming older. These immigrants will soon begin to retire and will shortly become a larger proportion of the elderly population. During the next twenty years, for example, the fastest growing elderly ethnic groups will be Chinese, South Asians, and persons from the Middle East. This will diversify our elderly population, with implications for the types of elderly services needed.

Social and economic conditions for immigrants in Canada have changed in recent years, with slower economic growth and poorer employment opportunities. We need to improve our understanding of these changing conditions for immigrants, and how immigrants have progressed in recent years. Because the success of the children of immigrants (the second immigrant generation) is so important for the future of Canadian society, it would be especially useful to know more about how immigrant and second-generation youth do after leaving school and entering the labour force.

\section{Life cycle changes}

The study of life cycle changes was originally developed by demographers to examine key transitions in individual lives, such as childhood education, becoming married or a parent, and retirement. Marshall and Mueller (2003) identified five policy domains where life cycle changes need to be considered: (1) education, the transition to employment, and lifelong learning; (2) family, and the relationship between work and family; (3) work-to-retirement transitions; (4) income security in the later years; and (5) intergenerational relations and social cohesion. In relation to the issue of peak global population, items 1,3, and 5 are the most likely to be affected, as discussed in the specific section on life cycle changes.

\section{Families}

Several interrelated issues deserve emphasis. First, early childhood education is important for overcoming difficulties faced by disadvantaged children and in order to support parental involvement in paid employment. Yet, we lack a good understanding of the role of different types of childhood education, including the costs and features of these programs, on lifetime development for children. Nor do we fully understand the relationship of childhood education and parental employment.

It is clear that societies which promote more family flexibility — as indicated by gender roles, family types, and types of sexual unions-encourage more young people to form longer-term relationships and partnerships, and to create families. This is an area where we need a better understanding of the role of family flexibility and such other factors as housing and urban geography on family formation. For example, it seems that housing and urban geography are important for accommodating the needs of families who have children and parents who have employment.

As the costs of children have increased, there are pressures on low and middle-income families, with the potential for delayed or reduced childbearing. This is a topic that requires further study from demographers.

Immigrant families provide a challenge for our understanding of several family-related issues. Foreign-born families in Canada have lower fertility than foreign-born families in other countries, 
such as the United States. While the reasons are not clear, factors such as Canada's selective immigration policy and origin countries of immigrants are likely implicated. Children of immigrants generally do well in schooling, although whether this educational success translates into commensurate occupational achievement and income remains an open question. In addition, immigrant families are aging, along with other Canadian families, yet current knowledge of aging immigrant families is quite sparse.

\section{Labour and migration in Arctic, rural, and remote communities}

Many professionals are becoming older and are likely to move after retirement. Recruitment and retention of replacement workers with technical and professional skills, especially in health care, will be a critical challenge in future years.

\section{Population and the environment}

The effect of population on the environment is perhaps the single issue most apparent to the public. Canada's success in reducing its share of greenhouse gases is more dependent than other countries on resource and energy extraction. A fundamental debate involves biologists and environmentalists who view resource limits as critical to dealing with environmental problems, while economists and other social scientists are more likely to stress that innovation and new technology offer the chance to deal with current and future environmental problems. Our understanding of population and the environment requires improved collaboration between natural and social scientists. As global population peaks and population growth slackens, the pressure of population numbers on the environment weakens. But, economic growth and new technology will continue, and will present environmental challenges in spite of slower population growth.

\section{Global Peak Population: Expanding our knowledge base}

Meeting future challenges also requires improving our knowledge base. We highlight three areas where greater efforts are needed.

First, at present we lack knowledge about a variety of important trends and processes in Canada's labour market. It is peculiar, for example, that we debate whether there are labour shortages or not; new studies are needed to understand better whether perceived labour shortages are due to a general shortage of workers, a lack of workers with specific skills and experience, or other factors. We especially need knowledge about employment over the lifetime for university graduates and recent immigrants, including a better understanding of the duration and intensity of employment, and the link between university studies and employment requirements. Such information would inform public discussion about employment by type of education, about the level of short-term unemployment, underemployment related to undesired over-qualification, and part-time employment, and the adaptation of immigrants based on their credentials and previous job experience.

Second, some important data sources, such as national data on marriages and divorces, have been lost in recent years. In addition, we need to improve or start new data sources on a number of topics, such as family dynamics and interrelationships with childbearing, employment, and early childhood education; elderly immigrants and their living arrangements, retirement income, and family support; lifetime data on taxes, health care, pensions, and social welfare benefits; and better data on northern populations. 
Third, collaborative research on population and the environment is needed to better understand the interrelationships of local and national population growth and its environmental effects. In particular, social scientists need to become more involved with the study of the energetics of Canadian society.

\section{References}

Davis, K. 1963. The theory of change and response in modern demographic history. Population Index 29(October):345-66.

Kirk, D. 1996. Demographic transition theory. Population Studies 50:361-87.

Marshall, V.W., and M.M. Mueller. 2003. Theoretical roots of the life course perspective, Chapter 1 in Social Dynamics of the Life Course: Transitions, Institutions, and Interrelations, edited by W.R. Heinz and V.W. Marshall. New York: Walter de Gruyter.

Statistics Canada. 2013. Immigration and Ethnocultural Diversity in Canada: National Household Survey, 2011. Cat. No. 99-010-X2011001. Ottawa: Ministry of Industry.

- 2015. Population Projections for Canada (2013 to 2063), Provinces and Territories (2013 to 2038). National Population Projections team (N. Bohnert, J. Chagnon, and P. Dion). Ottawa: Ministry of Industry.

United Nations. 2017. World Population Prospects: The 2017 Revision. Volume I: Comprehensive Tables. New York: United Nations Department of Economic and Social Affairs, Population Division. 